\title{
Primary Mantle Cell Lymphoma of Pancreas- Rare Case
}

Praveen Babu Jonnalagadda ${ }^{1 *}$, Dr. Sankar Subramanian, MS, $\mathrm{Mch}^{2}$, Dr. Suresh Kumar P, MS, Mch ${ }^{3}$, Dr. Chinni Vikram Asokan, $\mathrm{MS}^{4}$

\footnotetext{
${ }^{1,4}$ Mch Resident, ${ }^{2}$ Professor and Head, ${ }^{3}$ Assistant Professor, Department of Surgical Gastroenterology, Sri Ramachandra Institute of higher education and Research, Porur, Chennai, Tamil Nadu
}

DOI: $10.36347 /$ sjmcr.2022.v10i01.003

| Received: 05.12.2021 | Accepted: 09.01.2022 | Published: 14.01.2022

*Corresponding author: Praveen Babu Jonnalagadda

Mch Resident, Department of Surgical Gastroenterology, Sri Ramachandra Institute of higher education and Research, Porur, Chennai, Tamil Nadu

Abstract Case Report

Primary pancreatic lymphomas (PPLs) are rare cancers that have a complicated histological classification. Sufficient sample volumes are required to adequately diagnose and decide the best treatment for PPLs. A 54-year-old man with primary pancreatic mantle cell lymphoma is described in this case study. Percutaneous biopsy was used to extract histological samples. Atypical small to medium round cells predominated in the tumor cells, which had diffuse CD20 and cyclin D1 positivity. Chemotherapy was used on the patient, and he went into complete remission as a result. Because it provides adequate samples for testing, a percutaneous biopsy could be a valuable and safe diagnostic tool for PPLs.

Keywords: Primary pancreatic lymphoma, Endoscopic ultrasound-guided fine-needle aspiration, Pancreatic tumor. Copyright $(\mathcal{2 0 2 2}$ The Author(s): This is an open-access article distributed under the terms of the Creative Commons Attribution 4.0 International License (CC BY-NC 4.0) which permits unrestricted use, distribution, and reproduction in any medium for non-commercial use provided the original author and source are credited.

\section{INTRODUCTION}

Only about $2 \%$ of all extranodal lymphomas and 0.5 percent of all pancreatic masses are primary pancreatic lymphomas (PPLs) [1]. A PPL is a lymphomatous mass that is localized to the pancreas and manifests as a big bulky mass in the pancreatic head [2]. In 44 individuals, the median diameter of PPLs was $7.9 \mathrm{~cm}$ (range $2.4-13.9 \mathrm{~cm}$ ) according to a prior study [3]. Chemotherapy for PPLs has a high complete response rate [3], but proper pathological diagnosis necessitates a large sample size. Tumor biopsy by laparotomy obtains sufficient samples, however, it is invasive.

Despite the fact that endoscopic ultrasoundguided fine-needle aspiration (EUS-FNA) is a less invasive way for extracting tissue from pancreatic tumours [4], its effectiveness in identifying PPLs is questionable. We present a case of pancreatic mantle cell lymphoma in which the use of Ultrasound guided Percutaneous biopsy helped to make a correct diagnosis and choose an effective treatment.

\section{Case Presentation}

A 52-year-old man with abdominal pain and non-bilious vomiting presented to the outpatient department. Over the course of two weeks, he noticed greater fatigue and an unintended weight loss of $5 \mathrm{~kg}$. He also mentioned postprandial fullness and a lack of appetite. There was no recent foreign trip history, risky sexual behaviour, animal contact, or drug use history. He had not started any new prescriptions in the previous six months and was not taking any herbal remedies.

Hypertension and Type 2 Diabetes Mellitus were among his medical issues. He was on amlodipine and metformin at the time. He had an unremarkable surgical history and no medication sensitivities. He had a 5-pack-year smoking habit but did not drink alcohol.

On closer observation, he appeared pale, but there was no sign of mental illness. Temperature $36.4^{\circ} \mathrm{C}$, blood pressure $120 / 90 \mathrm{~mm} \mathrm{Hg}$, heart rate 68 beats per minute, respiratory rate 14 , and oxygen saturation 98 percent on air were among his findings. Physical examination revealed minor epigastric soreness, which was categorised as a 4/10.

Acetaminophen helped him feel better. A soft, reducible little umbilical hernia (defect $1 \mathrm{~cm} x 1 \mathrm{~cm}$ ) was found. The results of the respiratory and cardiovascular exams were unremarkable. 


\section{Investigations}

Normal liver function tests were verified by laboratory tests. He had a negative hepatitis test.

With a lactate of $0.8 \mathrm{mmol} / \mathrm{L} \quad(0.5-2.1$ $\mathrm{mmol} / \mathrm{L})$ and a $\mathrm{pH}$ of 7.395 (7.32-7.48), his venous blood gas revealed normal physiology. Urinalysis was normal study. There was no evidence of air beneath the diaphragm on his chest radiograph.

A low attenuation mass in the body and tail of the pancreas measured $15 \mathrm{~cm} \times 15 \mathrm{~cm}$ on a CT scan of the abdomen and pelvis with intravenous contrast, which was suggestive for malignancy (Figure 1). The tumour engulfed the pancreas' distal body and tail, with no dilation of the pancreatic duct. There were no visible lymph nodes in the hilar, mediastinal, or axillary regions, nor were there any lung or localised destructive bone lesions. The liver, spleen, adrenal glands, gall bladder, kidneys, bladder, and prostate gland all appeared to be in good working order. The retroperitoneal and mesenteric nodes were found to be somewhat enlarged. Pancreatic cancer (adenocarcinoma, lymphoma) with nodal metastasis was among the possibilities. His tumour markers were normal (AFP, Ca 125, CA 19-9, and CEA).

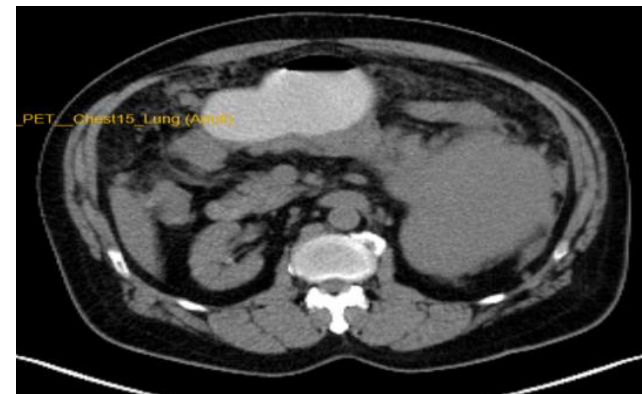

Fig 1: CT abdomen and pelvis with intravenous contrast showed large low attenuation mass in body and tail of pancreas measuring $15 \mathrm{~cm} \times 15 \mathrm{~cm}$

Using fluorine-18-fluorodeoxyglucose (FDG) positron emission tomography (PET)-CT, FDG uptake was found throughout the mass in the distal body and tail of the pancreas (maximum standardised uptake value: 11.93) (Fig 2).

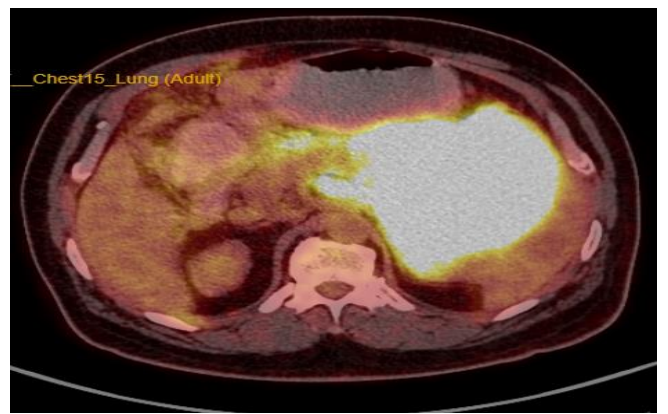

Fig 2: FDG PET-CT images. FDG PET-CT showed FDG uptake in the whole pancreatic lesion (SUV max of 11.93). FDG, fluorine18-fluorodeoxyglucose; PET-CT, positron emission tomography/computed tomography; SUVmax, maximum standard uptake value
Ultrasound guided Percutaneous biopsy with a 18-gauge needle was performed to assess the pancreatic tail mass.

The tumor cells were mostly made up of atypical small to medium round cells with a high nuclear/cytoplasmic ratio, with no Hodgkin or ReedSternberg cells (Fig 3 \& 4). Immunohistochemically (Table 1), the lesional cells are positive for CD20 (Fig 11), Cyclin D1 (Fig 6), CD45 (Fig 5), CD5 (Fig 7) and Bcl-2(Fig 5). They are negative for CD3 (Fig 8), INSM1, Panck, CD10, MUM1, Bcl-6. Ki- 67 (Fig 10) displays a proliferation index $>60 \%$. These morphologic and immunohistochemical features are diagnostic for Mantle cell lymphoma.

\section{Table 1}

\begin{tabular}{|l|l|}
\hline Antibody & Result \\
\hline C20 & Positive in lesional cells \\
\hline CD45 & Positive in lesional cells \\
\hline CD3 & Positive in Background T cells \\
\hline CD5 & Positive in lesional cells \\
\hline BCL2 & Positive in lesional cells \\
\hline CYCLIN D1 & Positive in lesional cells \\
\hline INSM1 & Negative in lesional cells \\
\hline Panck & Negative in lesional cells \\
\hline CD10 & Negative in lesional cells \\
\hline MUM1 & Negative in lesional cells \\
\hline BCL6 & Negative in lesional cells \\
\hline Ki 67\% & $>60 \%$ Positive \\
\hline
\end{tabular}

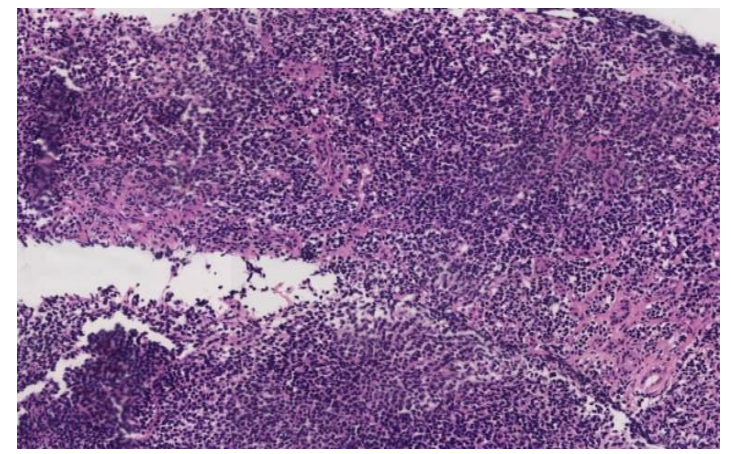

Fig 3: Large lymphoid cells with frequent mitotic figures and aoptotic bodies. Low power, $\mathbf{x 1 0}$

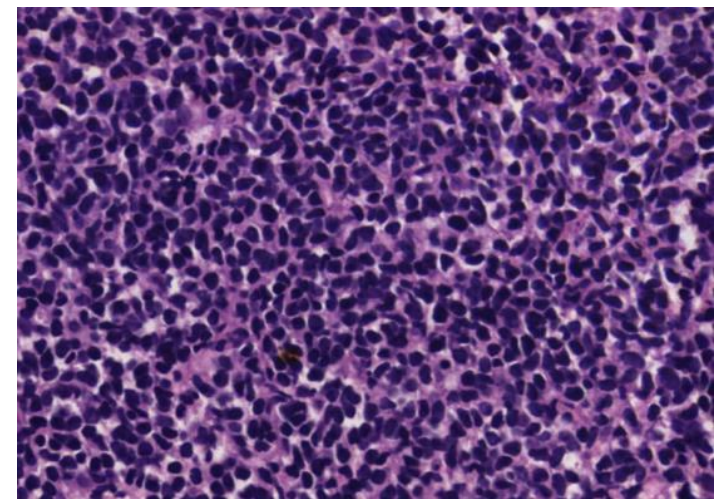

Fig 4: Large lymphoid cells with frequent mitotic figures and aoptotic bodies. High power, $x$ 
Praveen Babu Jonnalagadda et al., Sch J Med Case Rep, Jan, 2022; 10(1): 12-16

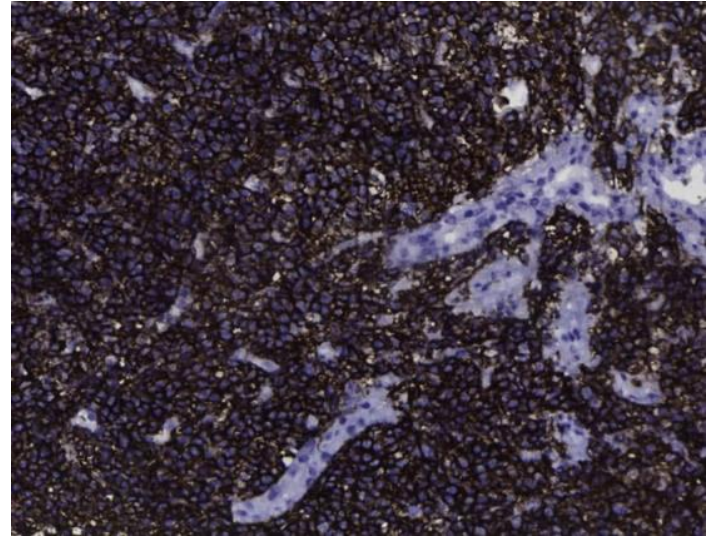

Fig 5: CD45 Positive

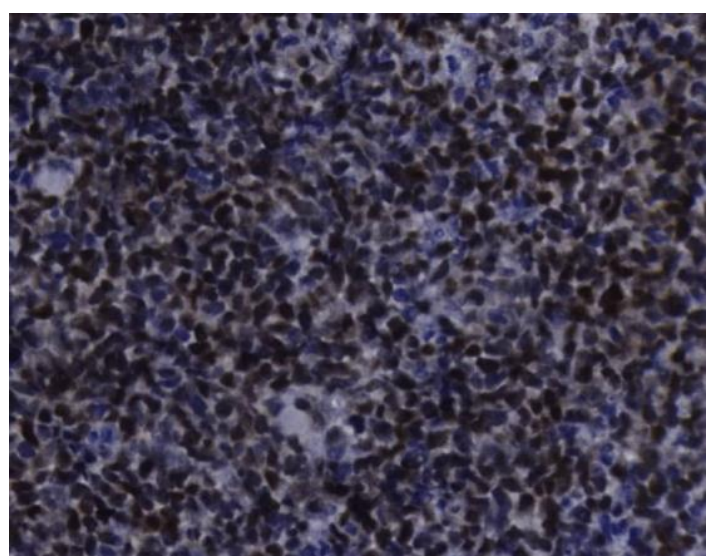

Fig 6: CYCLIN D1 Positive

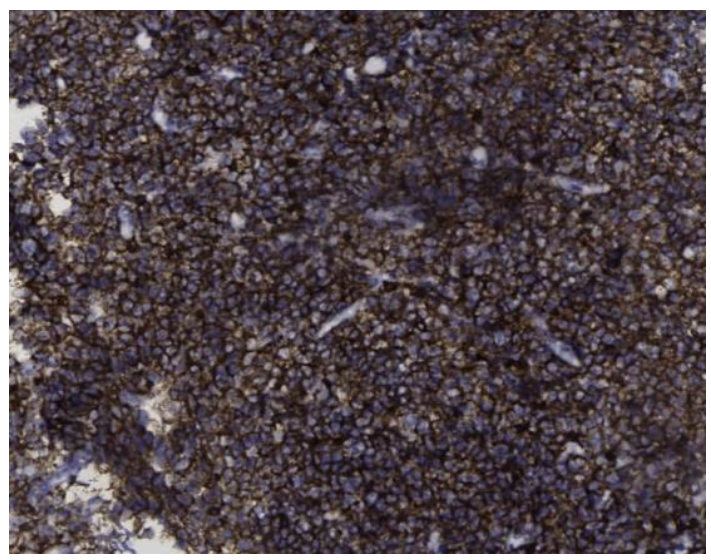

Fig 7: CD5 Positive

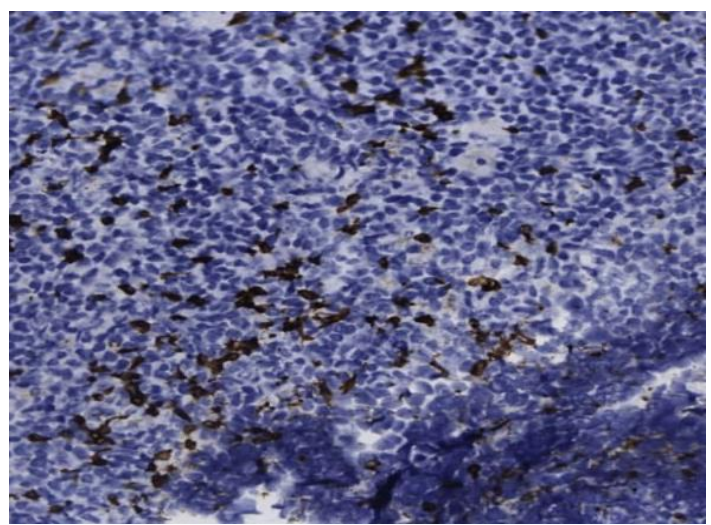

Fig 8: CD3 Positive Iin background T cells

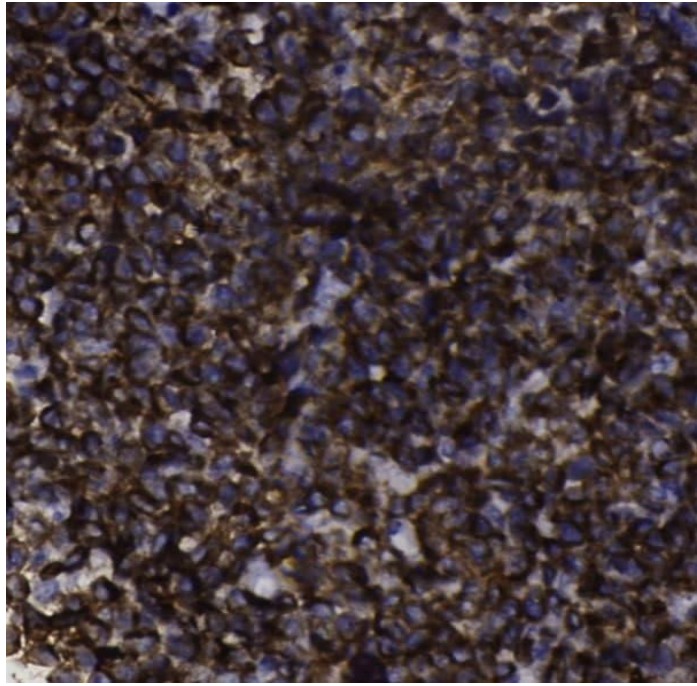

Fig 9: BCL2 Positive CD5

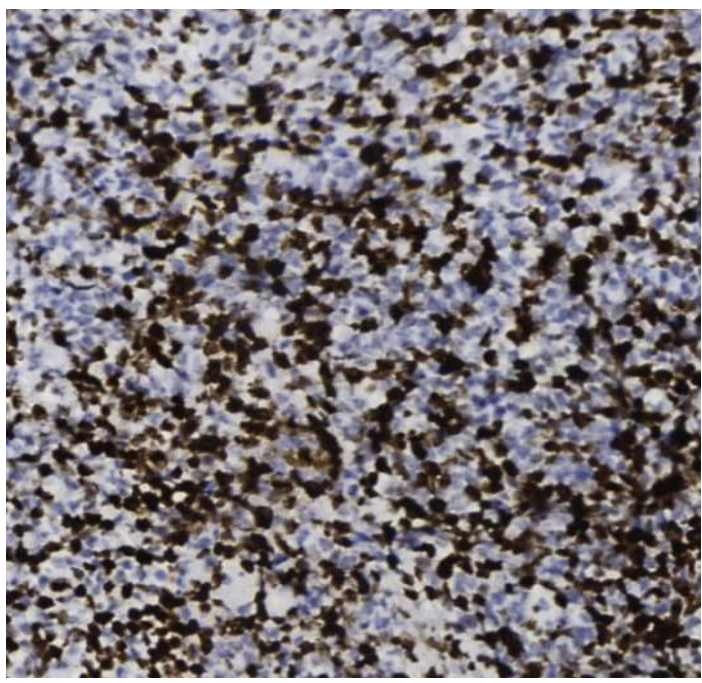

Fig 10: KI67 >60\% Positive

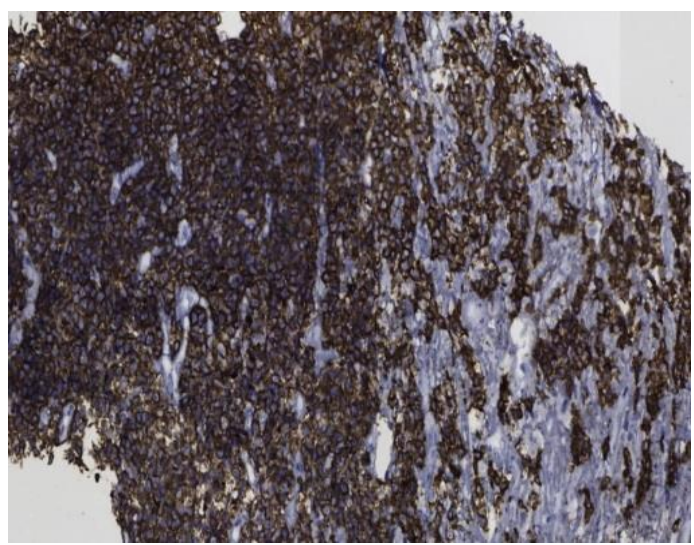

Fig 11: CD 20 immunostain showing B cells

According to the International Prognostic Index, these findings led to a final diagnosis of mantle cell lymphoma. He was in complete remission after 9 months of treatment with rituximab, pirarubicin, cyclophosphamide, vincristine, and prednisolone ( $\mathrm{R}$ THP-COP). PET-CT demonstrated FDG uptake in the pancreas and para-aortic lymph nodes one year and nine months after first-line treatment was stopped, indicating 
recurrence. He attained complete remission again after receiving the rituximab-bendamustine combo as a second-line treatment. The patient was doing well two years after starting first-line treatment and had no recurrence.

\section{DISCUSSION}

We provide a case of a patient who achieved long-term survival after chemotherapy after receiving an accurate pathological diagnosis of mantle cell lymphoma using ultrasound guided percutaneous biopsy. Because the head of the pancreas has more lymphoid tissues [7], a PPL is typically observed as a bulky mass in the head of the pancreas [6]. This is not the case in our situation.

Berhns et al., [8] proposed the following criteria for PPLs:

1. No palpable superficial lymphadenopathy.

2. No enlargement of mediastinal lymph nodes.

3. Normal leukocyte count.

4. At laparotomy, the pancreatic mass predominates with grossly involved lymph nodes confined to the peripancreatic region.

5. No hepatic or splenic involvement.

In combination with the MRI data, the patient allegedly met the criteria outlined above. A modestly contrast-enhanced tumor localized to the distal body and tail of the pancreas was visible on the contrastenhanced CT imaging. PET-CT scans, on the other hand, showed FDG buildup throughout the pancreatic tail lesion.

Clinical signs associated with a PPL were not observed in this case. Epigastric discomfort, nausea, exhaustion, and anorexia are recorded as clinical subjective symptoms of a PPL [3]. Severe fever, night sweats, and weight loss, which are common in other lymphomas (i.e., B signs), are uncommon in PPLs [9].

The current instance was mildly symptomatic and discovered during abdominal ultrasonography. The use of EUS-FNA in the differential diagnosis of pancreatic disorders has been proven to be effective [10]. Although a meta-analysis found that EUS-FNA had a high sensitivity (86.8\%) and specificity (95.8\%) for solid pancreatic tumors [11], it has been reported that histological examination with EUS-FNA is not suitable for the diagnosis of PPLs due to the difficulty in obtaining an adequate sample amount [12]. When compared to other pancreatic tumors, such as pancreatic ductal adenocarcinoma, a considerable volume of sample is required for the definite diagnosis of PPLs [13]. Flow cytometry and immunohistochemistry improve the diagnostic efficiency of PPLs when compared to cytology alone.

Although laparoscopic biopsies for the pathological analysis of PPLs have been undertaken, the laparoscopic examination is an intrusive technique since it requires general anesthesia and takes a long time (about 1-4 h) [14]. In our case, we were able to successfully perform immunostaining on specimens obtained through Ultrasound guided percutaneous biopsy, implying that it could be a useful and safe method for determining the definitive diagnosis of PPLs.

\section{CONCLUSiON}

We report a rare case of a primary pancreatic mantle cell lymphoma diagnosed via Ultrasound guided percutaneous biopsy, which provided a sufficient amount of sample for histological examination. Accurate histological findings helped to avoid unnecessary surgery and contribute to the selection of appropriate treatments. Ultrasound guided percutaneous biopsy could be a useful and safe diagnostic modality in pancreatic tail lesions where the differential diagnosis of a PPL is considered.

Statement of Ethics: Written informed consent was obtained from the patient for publication of this case report and any accompanying images.

Conflict of Interest Statement: The authors disclose no conflicts of interest.

Funding Sources: No specific funding has been received for this study.

Author Contributions: Study concept and design: Dr. Praveen Babu Jonnalagadda, Dr. Sankar Subramanian

Acquisition of data: Dr. Praveen Babu Jonnalagadda, Dr. Suresh Kumar P

Analysis and interpretation of data: Dr. Praveen Babu Jonnalagadda, Dr. Suresh Kumar P, Dr. Chinni Vikram Asokan

Drafting of the manuscript: Dr. Praveen Babu Jonnalagadda, Dr. Suresh Kumar P

Critical revision of the manuscript for important intellectual content: Dr. Praveen Babu Jonnalagadda, Dr. Suresh Kumar P

Final approval of the manuscript: all authors

\section{REFERENCES}

1. Teefey, S. A., Stephens, D. H., \& Sheedy, P. F. (1986). CT appearance of primary pancreatic lymphoma. Gastrointestinal radiology, 11(1), 4143.

2. Tuchek, J. M., De Jong, S. A., \& Pickleman, J. (1993). Diagnosis, surgical intervention, and prognosis of primary pancreatic lymphoma. The American surgeon, 59(8), 513-518. 
3. Sadot, E., Yahalom, J., Do, R. K. G., TeruyaFeldstein, J., Allen, P. J., Gönen, M., ... \& DeMatteo, R. P. (2015). Clinical features and outcome of primary pancreatic lymphoma. Annals of surgical oncology, 22(4), 1176-1184.

4. Mangiavillano, B., Sosa-Valencia, L., Deprez, P., Eisendrath, P., Robles-Medranda, C., Eusebi, L. H., ... \& Repici, A. (2020). Tissue acquisition and pancreatic masses: Which needle and which acquisition technique should be used?. Endoscopy International Open, 8(10), E1315-E1320.

5. Van Dongen, J. J. M., Langerak, A. W., Brüggemann, M., Evans, P. A. S., Hummel, M., Lavender, F. L., ... \& Macintyre, E. A. (2003). Design and standardization of PCR primers and protocols for detection of clonal immunoglobulin and $\mathrm{T}$-cell receptor gene recombinations in suspect lymphoproliferations: report of the BIOMED-2 Concerted Action BMH4-CT983936. Leukemia, 17(12), 2257-2317.

6. Rad, N., Khafaf, A., \& Alizadeh, A. H. M. (2017). Primary pancreatic lymphoma: what we need to know. Journal of gastrointestinal oncology, 8(4), 749-757.

7. Mishra, M. V., Keith, S. W., Shen, X., Ad, V. B., Champ, C. E., \& Biswas, T. (2013). Primary pancreatic lymphoma: a population-based analysis using the SEER program. American journal of clinical oncology, 36(1), 38-43.

8. Behrns, K. E., Sarr, M. G., \& Strickler, J. G. (1994). Pancreatic lymphoma: is it a surgical disease?. Pancreas, 9(5), 662-667.

9. Li, Z., Zhang, S., Vasdani, N., \& Castillo, E. (2012). Clues for diagnosing primary pancreatic lymphoma. Case reports in gastroenterology, 6(2), 438-445.

10. Lai, R., Stanley, M. W., Bardales, R., Linzie, B., \& Mallery, S. (2002). Endoscopic ultrasound-guided pancreatic duct aspiration: diagnostic yield and safety. Endoscopy, 34(09), 715-720.

11. Puli, S. R., Bechtold, M. L., Buxbaum, J. L., \& Eloubeidi, M. A. (2013). How good is endoscopic ultrasound-guided fine-needle aspiration in diagnosing the correct etiology for a solid pancreatic mass?: a meta-analysis and systematic review. Pancreas, 42(1), 20-26.

12. Thomas, T., Kaye, P. V., Ragunath, K., \& Aithal, G. (2009). Efficacy, safety, and predictive factors for a positive yield of EUS-guided Trucut biopsy: a large tertiary referral center experience. Official journal of the American College of Gastroenterology| ACG, 104(3), 584-591.

13. Johnson, E. A., Benson, M. E., Guda, N., Pfau, P. R., Frick, T. J., \& Gopal, D. V. (2014). Differentiating primary pancreatic lymphoma from adenocarcinoma using endoscopic ultrasound characteristics and flow cytometry: A case-control study. Endoscopic ultrasound, 3(4), 221-225.

14. Strasberg, S. M., Middleton, W. D., Teefey, S. A., McNevin, M. S., \& Drebin, J. A. (1999). Management of diagnostic dilemmas of the pancreas by ultrasonographically guided laparoscopic biopsy. Surgery, 126(4), 736-743.

15. Facciorusso, A., Buccino, R. V., \& Muscatiello, N. (2018). How to measure quality in endoscopic ultrasound. Annals of translational medicine, 6(13), 266. 